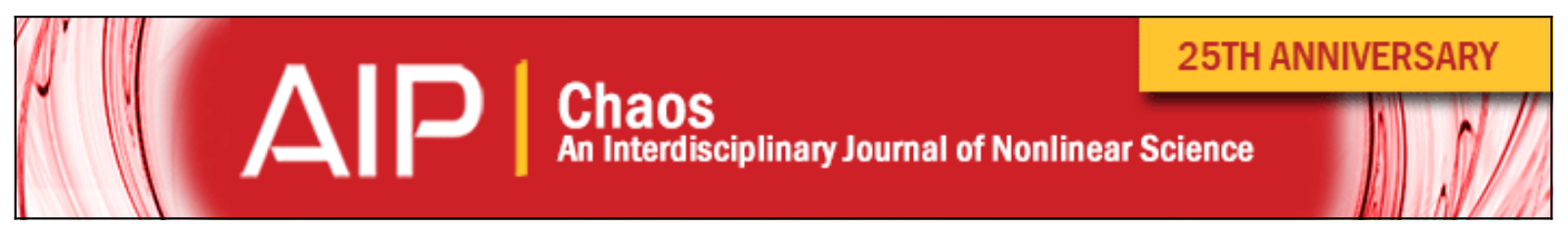

\title{
Measuring the hierarchy of feedforward networks
}

Bernat Corominas-Murtra, Carlos Rodríguez-Caso, Joaquín Goñi, and Ricard Solé

Citation: Chaos 21, 016108 (2011); doi: 10.1063/1.3562548

View online: http://dx.doi.org/10.1063/1.3562548

View Table of Contents: http://scitation.aip.org/content/aip/journal/chaos/21/1?ver=pdfcov

Published by the AIP Publishing

\section{Articles you may be interested in}

The estimation of neurotransmitter release probability in feedforward neuronal network based on adaptive synchronization

Chaos 23, 013109 (2013); 10.1063/1.4775757

Simultaneous fitting of a potential-energy surface and its corresponding force fields using feedforward neural networks

J. Chem. Phys. 130, 134101 (2009); 10.1063/1.3095491

Ab initio potential-energy surfaces for complex, multichannel systems using modified novelty sampling and feedforward neural networks

J. Chem. Phys. 122, 084104 (2005); 10.1063/1.1850458

Complex Networks and Socioeconomic Applications

AIP Conf. Proc. 661, 253 (2003); 10.1063/1.1571319

Isotropic vector hysteresis modeling with feed-forward neural networks

J. Appl. Phys. 91, 8322 (2002); 10.1063/1.1456400

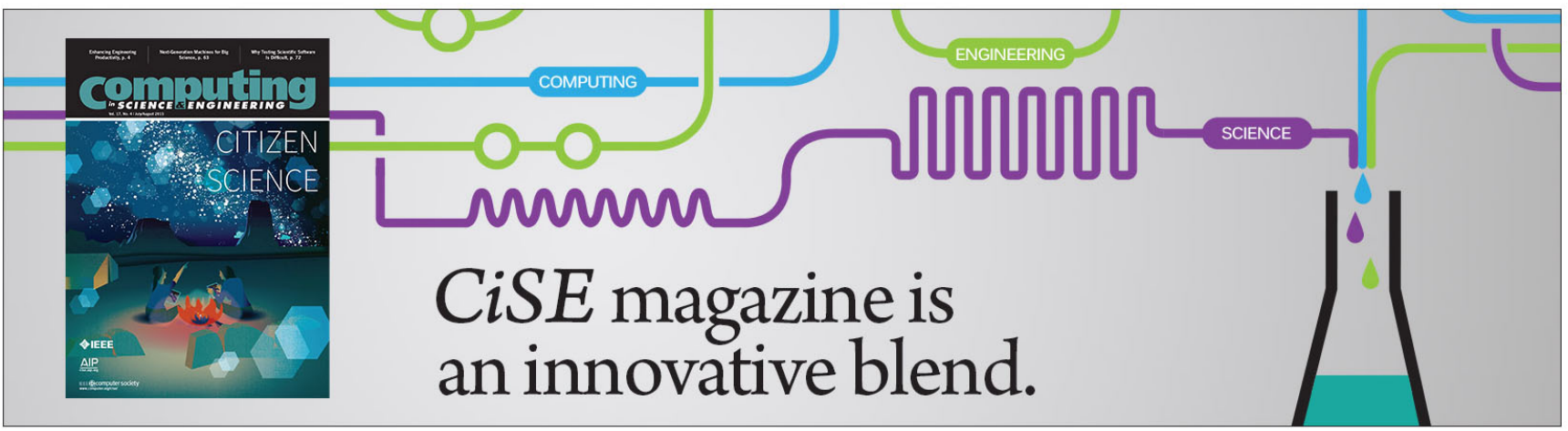




\title{
Measuring the hierarchy of feedforward networks
}

\author{
Bernat Corominas-Murtra, ${ }^{1,2, a)}$ Carlos Rodríguez-Caso, ${ }^{1,2, b)}$ Joaquín Goñi, $^{3}$ \\ and Ricard Solé ${ }^{1,2,4}$ \\ ${ }^{1}$ ICREA-Complex Systems Lab, Universitat Pompeu Fabra, Dr. Aiguader 88, 08003 Barcelona, Spain \\ ${ }^{2}$ Institut de Biologia Evolutiva, CSIC-UPF, Passeig Maritim de la Barceloneta, 37-49, 08003 Barcelona, \\ Spain \\ ${ }^{3}$ Neurosciences Department, Center for Applied Medical Research, University of Navarra, Pamplona, Spain \\ ${ }^{4}$ Santa Fe Institute, 1399 Hyde Park Road, Santa Fe, New Mexico 87501, USA
}

(Received 17 November 2010; accepted 16 February 2011; published online 29 March 2011)

\begin{abstract}
In this paper we explore the concept of hierarchy as a quantifiable descriptor of ordered structures, departing from the definition of three conditions to be satisfied for a hierarchical structure: order, predictability, and pyramidal structure. According to these principles, we define a hierarchical index taking concepts from graph and information theory. This estimator allows to quantify the hierarchical character of any system susceptible to be abstracted in a feedforward causal graph, i.e., a directed acyclic graph defined in a single connected structure. Our hierarchical index is a balance between this predictability and pyramidal condition by the definition of two entropies: one attending the onward flow and the other for the backward reversion. We show how this index allows to identify hierarchical, antihierarchical, and nonhierarchical structures. Our formalism reveals that departing from the defined conditions for a hierarchical structure, feedforward trees and the inverted tree graphs emerge as the only causal structures of maximal hierarchical and antihierarchical systems respectively. Conversely, null values of the hierarchical index are attributed to a number of different configuration networks; from linear chains, due to their lack of pyramid structure, to full-connected feedforward graphs where the diversity of onward pathways is canceled by the uncertainty (lack of predictability) when going backward. Some illustrative examples are provided for the distinction among these three types of hierarchical causal graphs.
\end{abstract}

(c) 2011 American Institute of Physics. [doi:10.1063/1.3562548]

The idea of hierarchy has been largely attributed to a disparate number of systems and, although easily perceived, its quantification is not a trivial issue. In this work, we quantify the hierarchy of a given causal structure with a feedforward structure. Starting with the representation of a system of causal relations as a graph, we define a nonheuristic measure of hierarchy having strong grounds on the principles of information theory. We depart from the definition of the conditions for a system to be considered perfectly hierarchical: a pyramidal structure with a completely predictable reversion of the causal flow. In this context, a hierarchy index is defined by weighting how far is a given feedforward structure from these conditions. As we shall see, structures that fully satisfy this property belong to a special class of trees. Our estimator allows to establish a quantitative criterion for the definition of hierarchic, nonhierarchic, and antihierarchic networks.

\section{INTRODUCTION}

The existence of some sort of hierarchical order is an apparently widespread feature of many complex systems, including gene $^{32}$ and human brain ${ }^{2,18}$ networks, ecosystems, ${ }^{11,19}$ social and urban structures, ${ }^{14}$ the Internet, ${ }^{30}$ or open-source commun-

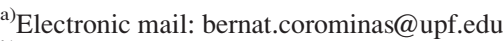

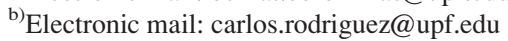

ities. ${ }^{29}$ The presence of such underlying order in the multiscale organization of complex systems is a long standing hypothe$\mathrm{sis}^{31}$ giving rise to the idea of hierarchy as a central conceptsee also Ref. 12. Although usually treated only in qualitative terms, some formal approaches to the problem have been proposed. The efforts toward a well-defined quantification of the hierarchical order have been improving by means of complex networks theory. As a key part of their organization, dedicated efforts have been made toward a proper identification of hierarchical trends. One outcome of these efforts has been a number of powerful and heuristic measures. 3,16,22,24,28,30

Often, a nested organization-formally identical to order, in set-theoretical terms ${ }^{15}$ - can be identified as the basis of hierarchical order. If we think in hierarchy in these terms, we might agree with Herbert Simon that "it is a commonplace that nature loves hierarchies"-cited in Ref. 20. Many examples belong to this picture. Within the context of matter organization, molecules are made of atoms, which result from the combination of elementary particles, some of which having also internal subunits (as quarks). Similarly, the relation of characteristic scales of organization by inclusion of one in another, like Chinese boxes or Matryoshka dolls, has been seen as a sort of hierarchical organization. Another relevant example is the case of fractal structures which naturally define a hierarchy of self-similar objects. Finally, within the context of taxonomy, spin glasses, or optimization theory, the use of ultrametricity has also allowed to define hierarchical order. ${ }^{21}$ 
Biological hierarchies have also evolved through time as part of a process that generates high-level entities out of nesting lower-level ones. ${ }^{717}$ However, taxonomic classification trees are probably the most obvious representation of a hierarchy based on inclusion. In biology, living beings are individuals grouped in taxa according to their characteristics. Starting from species, a nested hierarchy is defined where species belong to genera, which are included within families forming orders and so forth. Here, every organism is, in principle, unambiguously classified, and therefore no uncertainty can be associated to the process of classification.

Alternatively to the view of nestedness, a hierarchical organization can be defined in a structure of causal relations. Paradigmatic examples are the flowchart of a company or the chain of command in the army, where the authority concept defines a particular case of causal relation. Causality induces an asymmetrical link between two elements, and this asymmetry, either by inclusion or through any kind of causal relation, defines an order. We observe that any circular or symmetrical relation between two elements violates the concept of order, thereby intuitively loosing its hierarchical nature. From this perspective, any feedforward relation is potentially hierarchical. Such feedforward structures pervade a diverse range of phenomena and structures, from phylogenetic trees to river basins.

A common feature of most of the approaches mentioned above is that the notion of hierarchy is basically identified with the concept of order. Order is a well-defined concept in mathematics, ${ }^{15,27}$ but, is order enough to grasp the intuitive idea of hierarchy? Can we actually define what is hierarchy? Quoting Herbert Simon, ${ }^{26}$ (...) a hierarchical system-or hierarchy - can be defined in principle as a system that is composed of interrelated subsystems, each of the latter being also hierarchic in structure until the lowest scale is reached. ${ }^{33}$

This definition does not provide a clear formalization of hierarchy as a measurable feature, although it certainly grasps the intuitive idea of hierarchy. How can such a general measure be defined? It is reasonable to assume that we have a hierarchy if there is no ambiguity (or uncertainty) in the chain of command followed for any individual to the chief in the flowchart. We shall call this feature the definiteness condition. This is also valid for nested structures. One might think that this condition is justified by a single chain of command or in the case of matryoshka dolls. However, such structures are already defined within order theory as totally ordered [see Fig. 1(a)]. Order and hierarchy are closely related but they are not essentially the same. In this paper, we reserve the word hierarchy to designate a concept that goes beyond the definition of order. We argue that the difference stems from the fact that a hierarchical structure must also satisfy a pyramidal organization constraint—see Fig. 1(b). In other words, the lower the layer of organization, the larger the number of entities it contains. But, what happens when this pyramid structure is inverted? Intuitively, they would not be hierarchical but antihierarchical. ${ }^{34}$ In this work we show how information theory naturally provides the suitable framework to characterize hierarchy in causal structures. Within this theoretical apparatus, we provide the rigorous definition of the hierarchy index for causal structures, and how it is applied in some illustrative examples establishing a distinction among hierarchical, nonhierarchical, and antihierarchical.

\section{DIRECTED GRAPHS, ORDERED GRAPHS, AND CAUSAL GRAPHS}

In this section, we present the basic theoretical background used in this paper, grounded both in order and graph theories. At the end of this section, we will formally define the causal graph, the key concept of this theoretical framework where the proposed hierarchy measures are applied.

\section{A. Basic concepts of order}

Hierarchy is undoubtedly tied to order. This is why we make a brief review of order theory highlighting some features that have been commonly attributed to hierarchy. The first task is to define an ordered pair between two elements $a_{k}, a_{j}$ of a given set $A$, to be written as $a_{k}>a_{j},\left\langle a_{k}, a_{j}\right\rangle$, or, in a formally equivalent way

$$
\left\langle a_{k}, a_{j}\right\rangle=\left\{\left\{a_{k}\right\},\left\{a_{k}, a_{j}\right\}\right\} .
$$

This latter formalization explicitly defines order from an inclusion relation. ${ }^{15}$ This immediately connects to the standard views of hierarchical systems, as we already mentioned, in which the inclusion relations are considered essential. Having defined an ordered pair, we define an order relation. Let $A=\left\{a_{1}, \ldots, a_{n},\right\}$ be a countable and finite set and $\mathcal{R} \in A \times A$ a relation. Such a relation is an order relation - rigorously speaking, a strict partial order — if the following condition holds:

(i) $\left\langle a_{k}, a_{k}\right\rangle \notin \mathcal{R}$,

(ii) $\left(\left\langle a_{i}, a_{k}\right\rangle \in \mathcal{R}\right) \Rightarrow\left(\left\langle a_{k}, a_{i}\right\rangle \notin \mathcal{R}\right)$,

(iii) $\left(\left\langle a_{i}, a_{k}\right\rangle \in \mathcal{R} \wedge\left\langle a_{k}, a_{j}\right\rangle \in \mathcal{R}\right) \Rightarrow\left(\left\langle a_{i}, a_{j}\right\rangle \in \mathcal{R}\right)$.

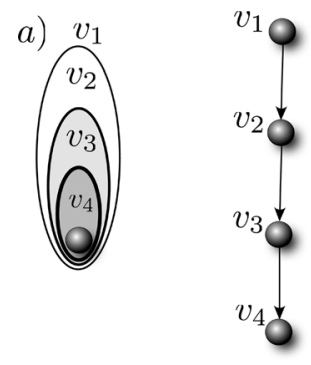

b)

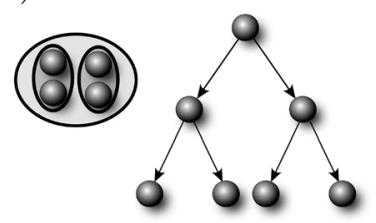

c)

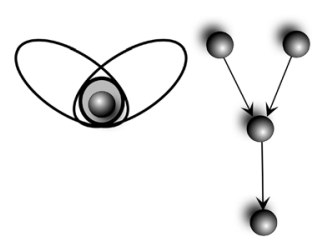

FIG. 1. Order and hierarchy. (a) Total or complete order either defined by inclusion displaying nested ensembles, $v_{1} \subset v_{2} \subset v_{3} \subset v_{4}$ or in terms of the order relation $v_{1}>v_{2}>v_{3}>v_{4}$ (left). The direct or immediate relation depicted by its causal graph (right). (b) The ideal hierarchy structure assumed in this work represented by its nested organization (left) and its causal graph (right). (c) An example of a partial ordered defined by inclusion (left) and its respective causal graph (right). 
We finally define two subsets of $A$ from the definition of order relation which will be useful to characterize the kind of structures studied in this paper. The set of maximal elements of $A$, to be written as $M \subset A$, is defined as

$$
M=\left\{a_{k} \in A: \nexists a_{j} \in A:\left\langle a_{j}, a_{k}\right\rangle \in \mathcal{R}\right\} .
$$

Similarly, the set of minimal elements, to be written as $\mu \subset A$ is defined as

$$
\mu=\left\{a_{k} \in A: \nexists a_{j} \in A:\left\langle a_{k}, a_{j}\right\rangle \in \mathcal{R}\right\} .
$$

\section{B. Basic concepts of directed acyclic graphs}

Let $\mathcal{G}(V, E)$ be a directed graph, being $V=\left\{v_{1}, \ldots, v_{n}\right\}$, $>|V|=n$ the set of nodes and $E=\left\{\left\langle v_{k}, v_{i}\right\rangle, \ldots,\left\langle v_{j}, v_{l}\right\rangle\right\}$ the set of arcs-where the order, $\left\langle v_{k}, v_{i}\right\rangle$, implies that there is an arc in the following direction: $v_{k} \rightarrow v_{i}$. Given a node $v_{i} \in V$, the number of outgoing links, to be written as $k_{\text {out }}\left(v_{i}\right)$, is called the out-degree of $v_{i}$, and the number of ingoing links of $v_{i}$ is called the in-degree of $v_{i}$, written as $k_{\text {in }}\left(v_{i}\right)$. The adjacency matrix of a given graph $\mathcal{G}, \mathbf{A}(\mathcal{G})$ is defined as $A_{i j}(\mathcal{G})=1 \leftrightarrow\left\langle v_{i}, v_{j}\right\rangle \in E ; \quad$ and $\quad A_{i j}(\mathcal{G})=0 \quad$ otherwise. Through the adjacency matrix, $k_{\text {in }}$ and $k_{\text {out }}$ are computed as

$$
k_{\text {in }}\left(v_{i}\right)=\sum_{j \leq n} A_{j i}(\mathcal{G}) \quad k_{\text {out }}\left(v_{i}\right)=\sum_{j \leq n} A_{i j}(\mathcal{G}) .
$$

Furthermore, we will use the known relation between the $k$-th power of the adjacency matrix and the number of paths of length $k$ going from a given node $v_{i}$ to a given node $v_{j}$. Specifically,

$$
\left(\mathbf{A}^{k}(\mathcal{G})\right)_{i j}=(\overbrace{\mathbf{A}(\mathcal{G}) \times \cdots \times \mathbf{A}(\mathcal{G})}^{k \text { times }})_{i j}
$$

is the number of paths of length $k$ going from node $v_{i}$ to node $v_{j}{ }^{10}$

It is said that $v_{i}$ dominates $v_{k}$ if $\left\langle v_{i}, v_{k}\right\rangle \in E$. A feedforward or directed acyclic graph (DAG) is a directed graph characterized by the absence of cycles: If there is a directed path from $v_{i}$ to $v_{k}$ (i.e., there is a finite sequence $\left.\left\langle v_{i}, v_{j}\right\rangle,\left\langle v_{j}, v_{l}\right\rangle,\left\langle v_{l}, v_{s}\right\rangle, \ldots,\left\langle v_{m}, v_{k}\right\rangle \in E\right)$ then, there is no directed path from $v_{k}$ to $v_{i}$. Conversely, the matrix $\mathbf{A}^{T}(\mathcal{G})$ depicts a DAG with the same underlying structure but having all the arrows (and thus, the causal flow) inverted. The underlying graph of a given DAG $\mathcal{G}$, to be written as $\mathcal{G}_{u}$, is the undirected graph $\mathcal{G}^{u}\left(V, E^{u}\right)$ obtained by substituting all arcs of $E,\left\langle v_{i}, v_{k}\right\rangle,\left\langle v_{j}, v_{s}\right\rangle, \ldots$ by edges giving the set $E^{u}=\left\{v_{i}, v_{k}\right\},\left\{v_{j}, v_{s}\right\}, \ldots$ A DAG $\mathcal{G}$ is said to be connected if for any pair of nodes combination $v_{i}, v_{l} \in V$ there is a finite sequence of pairs having the following structure:

$$
\left\{v_{i}, v_{k}\right\},\left\{v_{k}, v_{j}\right\}, \ldots,\left\{v_{m}, v_{s}\right\},\left\{v_{s}, v_{l}\right\},
$$

being $\left\{v_{i}, v_{k}\right\},\left\{v_{k}, v_{j}\right\}, \ldots,\left\{v_{m}, v_{s}\right\},\left\{v_{s}, v_{l}\right\} \in E_{u}$. Given the acyclic nature of a DAG, one can find a finite value $L(\mathcal{G})$ as follows:

$$
L(\mathcal{G})=\max \left\{k:\left(\exists v_{i}, v_{j} \in V:\left(\mathbf{A}^{k}(\mathcal{G})\right)_{i j} \neq 0\right)\right\} .
$$

It is easy to see that $L(\mathcal{G})$ is the length of the longest path of the graph.

Borrowing concepts from order theory, ${ }^{27}$ we define the following set:

$$
M=\left\{v_{i} \in V: k_{\text {in }}\left(v_{i}\right)=0\right\},
$$

to be named the set of maximal nodes of $\mathcal{G}$, by which $|M|=m$. Additionally, one can define the set of nodes $\mu$ as

$$
\mu=\left\{v_{i} \in V: k_{\text {out }}\left(v_{i}\right)=0\right\}
$$

to be referred as the set of minimal nodes of $\mathcal{G}$.

The set of all paths $\pi_{1}, \ldots, \pi_{s}, s \geq|E|$, from $M$ to a given node $v_{i} \in \mu$ is indicated as $\Pi_{M \mu}(\mathcal{G})$. Given a node $v_{i} \in \mu$, the set of all paths from $M$ to $v_{i}$ is written as

$$
\Pi_{M \mu}\left(v_{i}\right) \subseteq \Pi_{M \mu}(\mathcal{G}) .
$$

Furthermore, we will define the set $v\left(\pi_{k}\right)$ as the set of all nodes participating in this path, except the maximal one. Conversely, the set $\tilde{v}\left(\pi_{k}\right)$ is the set of all nodes participating on this path, except the minimal one. Attending to the node relations, depicted by the arrows, and due to the acyclic property, at least one node ordering can be defined, establishing a natural link between order theory and DAGs. This order is achieved by labeling all the nodes with sequential natural numbers and obtaining a configuration such that

$$
\left(\forall\left\langle v_{i}, v_{j}\right\rangle \in E\right)(i<j) .
$$

The existence of this labeling connects order relations with directed acyclic graphs.

Finally, throughout this paper, we reserve the word tree to refer to those graphs where all nodes excluding the maximal one have $k_{\text {in }}=1$ and all nodes except the minimal ones display $k_{\text {out }}>1$. Therefore, we distinguish between chains (all nodes with $k_{\text {out }}=1$ excluding the minimal one) and trees.

\section{Causal graphs}

In a causal graph, we consider only immediate relations between elements, i.e., two elements are causally related if there exists just one cause-effect event relating them. We explicitly neglect those relations between nodes which can only be derived by transitivity. A causal relation can be illustrated by genetic inheritance in a genealogy. Offspring's characters come from its parents and indirectly from its grandparents. Therefore, no direct causal relation can be defined between grandparents and grandsons. However, it is true that grandparents indirectly determine the characters of grandsons due to the transitive nature of the genetic relations.

In this work we will restrict the use of the term causal relation to refer to direct relations such as parents-sons relations, as described in the above example. A causal graph $\mathcal{G}(V, E)$ is a directed graph where $V$ are the elements of a set (the members of a family, in the above described example) and $E$ are the causal relations that can be defined between the members of $V$. In this work, we restrict the term causal graphs to graphs being acyclic (i.e., DAGs) and connected. The former property avoids conflicts in the definition of the 
causal flow. The latter property assumes that two nonconnected causal structures have no relation among them, and therefore must be considered as two independent systems. Hereafter, we will refer to the set of paths $\Pi_{M \mu}(\mathcal{G})$ as the set of causal paths.

\section{THE CONCEPTUAL BACKGROUND OF HIERARCHY}

In this section, we propose the basis for a rigorous evaluation of hierarchy. We begin by defining the features of what we consider as the perfect hierarchical structure. As shown below, our proposed definition of hierarchy matches with an ordered structure with special features, thereby making an explicit difference between order and hierarchy. Therefore, we reserve the term hierarchy to refer to a special class of order. Within the framework of graph theory, the required conditions naturally match those displayed by a treelike feedforward graph. Then, as we shall see, the estimator we propose identifies the feedforward tree topology as perfectly hierarchical. The main point of the section is devoted to the definition of a quantitative estimator of hierarchy based on two entropy measures that capture the intuitive ideas described in the introductory section: the definiteness and pyramidal organization condition. We stress that the forthcoming formalism applies only to the class of causal graphs.

\section{A. The starting point: Defining the perfect hierarchy}

We are going to refer to a system as perfectly hierarchical if it satisfies the following conditions. Let us consider a system depicted by a causal graph $\mathcal{G}(V, E)$. We say that this graph $\mathcal{G}$ will be perfectly hierarchical if the following two conditions hold:

1. Definiteness condition.-For every element $v_{k} \in V \backslash M$ there is only one element $v_{i} \in V, \quad v_{i} \neq v_{k}$ such that $\left\langle v_{i}, v_{k}\right\rangle \in E$. A straightforward consequence of this condition is that

$$
m=1 \text {. }
$$

2. Pyramidal condition.-There is a partition $W=\left\{\omega_{1}, \ldots\right.$, $\left.\omega_{m}\right\}$ of the set $V$, i.e.,

$$
V=\bigcup_{W} \omega_{i} \quad \forall \omega_{i}, \omega_{k} \in W, \omega_{i} \cap \omega_{k}=\emptyset
$$

by which

$$
\left(\forall\left\langle v_{i}, v_{\ell}\right\rangle \in E\right)\left(v_{i} \in \omega_{j}\right) \Rightarrow\left(k_{\text {out }}\left(v_{i}\right)>1\right) \wedge\left(v_{\ell} \in \omega_{j+1}\right) .
$$

A direct consequence of the above is that

$$
\left|\omega_{1}\right|<\left|\omega_{2}\right|<\cdots<\left|\omega_{|W|}\right|,
$$

which reflects the pyramidal structure of the graph. A measure of hierarchy must properly weight the deviations of the studied graph from the above requirements. One could add another condition by imposing that every node in a given layer dominates the same number of nodes contained in the next downstream layer. In formal terms, this implies that, in addition to the above conditions, we can add a third one, namely,
3. Symmetry condition. It is established by means of

$$
\left(\forall v_{i}, v_{\ell} \in \omega_{j}\right)\left(k_{\text {out }}\left(v_{i}\right)=k\right) \Rightarrow\left(k_{\text {out }}\left(v_{\ell}\right)=k\right),
$$

which actually corresponds to a so-called complete $k$-ary tree. ${ }^{10}$ Therefore, in those cases where symmetry is considered as an inherent feature of ideal hierarchy, deviations from symmetrical configurations must also be taken into account in our quantitative approximation.

Let us summarize the above statements (1), (2), and (3) and their consequences. The so-called definiteness condition implies that there is no uncertainty in identifying the premise of a given causal relation, i.e., the node that immediately governs a given node. Taking into account the definition of causal graph (essentially, a connected DAG), this first statement restricts to tree structures, the number of candidates for perfectly hierarchical structures. Note that these trees-including linear chains-have a single root node, i.e., $m=1$. The pyramidal condition rules out from the set of perfectly hierarchical structures those DAGs having linear chains in their structure. In other words, nodes displaying $k_{\text {in }}=k_{\text {out }}=1$ are not allowed. We observe that, according to the pyramidal condition,

$$
\left|\omega_{i}\right| \leq \frac{\left|\omega_{i+1}\right|}{2} .
$$

Therefore, it is straightforward to conclude that the simplest representation of an ideal hierarchical structure is a binary tree-see Fig. 2(a) — in which the above inequality becomes equality for all successive layers. This is consistent with standard graph theory and the definition of perfect binary tree. Finally, the symmetry condition, optional for our definition of hierarchy, rules out those trees which are not perfectly symmetrical. Whereas trees shown in Fig. 2(a) and 2(b) can be considered hierarchical by virtue of conditions (1) and (2); condition (3) makes a distinction between them, being only perfectly hierarchical the first one.

As a final remark, let us note that one can build nonhierarchical and antihierarchical structures by simply violating some of the conditions we stated above, namely, the pyramidal condition alone-Figs. 2(e)—or both the definiteness and the pyramidal condition-see Fig. 2(d) and 2(f). It is easy to see that a quantitative estimator of hierarchy should account for limit cases and place as intermediate point structures such as the one depicted in 2(c).

\section{B. Topological richness and reversibility}

The above features describe the perfect hierarchical structure. In this section, we go further and we provide the basis for a definition of a hierarchical index of a causal graph grounded in the framework of information theory. This index provides a quantitative estimation of how far is a given causal graph from the conditions of a perfect hierarchy. 
In the following subsections, we will define two entropies for a causal graph, attending to the top-down and bottom-up observations of the causal graph according to the onward and backward flows in the graph. The aim of this mathematical formalism is to quantify the impact of the number of pathways in the causal graph. Specifically, we will consider the balance between the richness of causal paths (a top-down view) versus the uncertainty when going back reversing the causal flow (i.e., a bottom-up perspective). Then, attending to the direction of the flow we interpret the top-down view as a richness while the bottom-up as an uncertainty in terms of topological reversibility, as recently introduced in Ref. 4. Arguably, the larger is the number of

a)
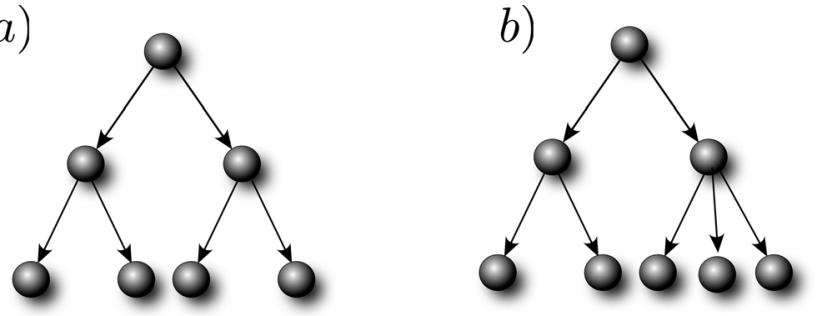

c)
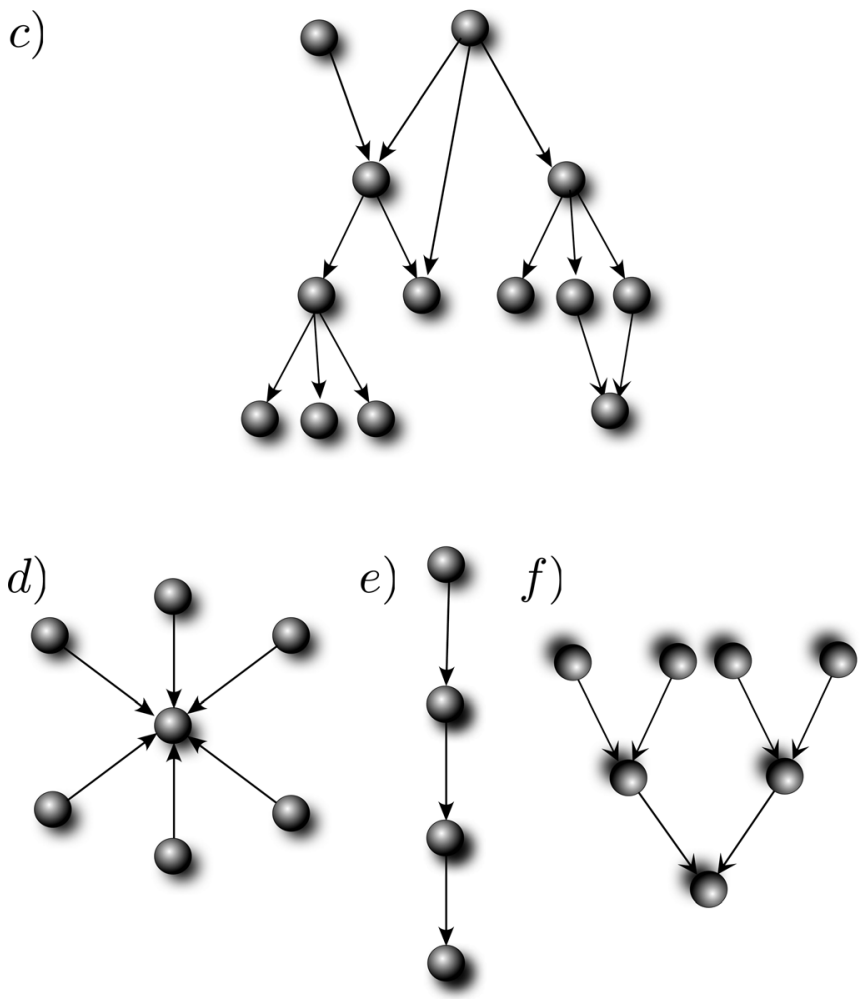

FIG. 2. Causal graphs intuitively capturing different degrees of hierarchy. (a) A symmetrical treelike causal graph showing the ideal hierarchical structure assumed in this work. (b) An asymmetrical treelike hierarchical graph. (c) A causal graph, with a pseudopyramidal condition violated by the presence of more than one maximal node and short-cuts between layers. Intuitively nonhierarchical and antihierarchical structures illustrated by (d) an antihierarchical star graph with $|V-1|$ maximals exhibiting and inverted pyramidal condition (note that inverting the arrows we would have a treelike graph), (e) an ordered but no hierarchical linear chain violating the pyramidal condition, and (f) an inverted treelike structure where no definiteness and pyramicity conditions are satisfied onward but completely satisfied backward. decisions going down, the higher is the richness of causal paths. Similarly, the larger the number of alternative pathways to climb up, the larger will be the uncertainty in recovering the causal flow. In the following subsections, we will explore, within the framework of information theory, the relationship between diversity and uncertainty and their impact in the fulfillment of the hierarchy conditions. We begin this section with a brief revision of the core concepts of information theory, to be used as our theoretical framework.

According to classical information theory, ${ }^{1,6,13,25}$ let us consider a system $S$ with $n$ possible states, whose occurrences are governed by a random variable $X$ with an associated probability mass function formed by $p_{1}, \ldots, p_{n}$. According to the standard formalization, the uncertainty or entropy associated to $X$, to be written as $H(X)$, is

$$
H(X)=-\sum_{i \leq n} p_{i} \log p_{i}
$$

which is actually an average of $\log (1 / p(X))$ among all events of $S$, namely, $H(X)=\langle\log (1 / p(X))\rangle$, where $\langle\ldots\rangle$ is the expectation or average of the random quantity between parentheses. Analogously, we can define the conditional entropy. Given another system $S^{\prime}$ containing $n^{\prime}$ values or choices, whose behavior is governed by a random variable $Y$, let $\mathbb{P}\left(s_{i}^{\prime} \mid s_{j}\right)$ be the conditional probability of obtaining $Y=s_{i}^{\prime} \in S^{\prime}$ if we already know $X=s_{j} \in S$. Then, the conditional entropy of $Y$ from $X$, to be written as $H(Y \mid X)$, is defined as

$$
H(Y \mid X)=-\sum_{j \leq n} p_{j} \sum_{i \leq n^{\prime}} \mathbb{P}\left(s_{i}^{\prime} \mid s_{j}\right) \log \mathbb{P}\left(s_{i}^{\prime} \mid s_{j}\right) .
$$

\section{Topological reversibility: Definiteness condition}

The first task is to study the degree of reversibility of causal paths, thereby considering the role of the definiteness condition. This will be evaluated by computing the uncertainty in reversing the process starting from a given node in $\mu$. The formalism used in this section is close to the one developed in Ref. 4.

We first proceed to define the probability distribution from which the entropy will be evaluated. Accordingly, the probability to choose a path $\pi_{k} \in \Pi_{M \mu}$ from node $v_{i} \in \mu$ by making a random decision at every crossing when reverting the causal flow is

$$
\mathbb{P}\left(\pi_{k} \mid v_{i}\right)=\prod_{v_{i} \in v\left(\pi_{k}\right)} \frac{1}{k_{\mathrm{in}}\left(v_{j}\right)} .
$$

The conditional entropy obtained when reverting the flow from $v_{i} \in \mu$ will be

$$
H\left(\mathcal{G} \mid v_{i}\right)=-\sum_{\pi_{k} \in \Pi\left(v_{i}\right)} \mathbb{P}\left(\pi_{k} \mid v_{i}\right) \log \mathbb{P}\left(\pi_{k} \mid v_{i}\right) .
$$

The overall uncertainty of $\mathcal{G}$, written as $H(\mathcal{G} \mid \mu)$, is computed by averaging $H$ over all minimal nodes, i.e, 


$$
\begin{aligned}
H(\mathcal{G} \mid \mu) & =\sum_{v_{i} \in \mu} q\left(v_{i}\right) H\left(\mathcal{G} \mid v_{i}\right) \\
& =-\sum_{v_{i} \in \mu} q\left(v_{i}\right) \sum_{\pi_{k} \in \Pi\left(v_{i}\right)} \mathbb{P}\left(\pi_{k} \mid v_{i}\right) \log \mathbb{P}\left(\pi_{k} \mid v_{i}\right) \\
& =\sum_{v_{i} \in \mu} q\left(v_{i}\right) \sum_{\pi_{k} \in \Pi\left(v_{i}\right)}\left[\sum_{v_{j} \in v\left(\pi_{k}\right)} \mathbb{P}\left(\pi_{k} \mid v_{i}\right) \log \left(k_{\text {in }}\left(v_{j}\right)\right)\right] \\
& =\sum_{v_{i} \in \mu} q\left(v_{i}\right) \sum_{v_{j} \in V\left(\Pi\left(v_{i}\right)\right.} \log \left(k_{\text {in }}\left(v_{j}\right)\right)\left[\sum_{\pi_{k}: v_{j} \in v\left(\pi_{k}\right)} \mathbb{P}\left(\pi_{k} \mid v_{i}\right)\right] \\
& =\sum_{v_{i} \in \mu} q\left(v_{i}\right) \sum_{v_{k} \in V \backslash M} \phi_{i k}(\mathcal{G}) \log k_{\text {in }}\left(v_{k}\right),
\end{aligned}
$$

where we assume, unless indicated

$$
\left(\forall v_{i} \in \mu\right) \quad q\left(v_{i}\right)=\frac{1}{|\mu|} .
$$

Instead of a vector, now we construct a $(n-m) \times(n-m)$ matrix, $\Phi(\mathcal{G})$ accounting for the combinatorics of paths and how they contribute to the computation of entropy

$$
[\Phi(\mathcal{G})]_{i j} \equiv \phi_{i j}(\mathcal{G})=\sum_{\pi_{k}: v_{j} \in v\left(\pi_{k}\right)} \mathbb{P}\left(\pi_{k} \mid v_{i}\right)
$$

This represents the probability to reach $v_{j}$ starting from $v_{i}$. Now we derive the general expression for $\Phi$. To compute the probability to reach a given node, we have to take into account the probability to follow a given path containing such a node, defined in Ref. 8. To rigorously connect it to the adjacency matrix, we first define an auxiliary, $(n-m) \times(n-m)$ matrix $\mathbf{B}(\mathcal{G})$, namely,

$$
B(\mathcal{G})_{i j}=\frac{A_{i j}(\mathcal{G})}{k_{\text {in }}\left(v_{i}\right)},
$$

where $v_{i}, v_{j} \in V \backslash M$. From this definition, we obtain the explicit dependency of $\Phi$ from the adjacency matrix, namely,

$$
\phi_{i j}(\mathcal{G})=\sum_{k \leq L(\mathcal{G})}\left(\left[\mathbf{B}^{T}\right]^{k}(\mathcal{G})\right)_{i j}
$$

and accordingly, we have

$$
\phi_{i i}(\mathcal{G})=\left(\left[\mathbf{B}^{T}\right]^{0}(\mathcal{G})\right)_{i i}=1 .
$$

Therefore, we already obtained the explicit form of such a conditional entropy, namely,

$$
H(\mathcal{G} \mid \mu)=\sum_{v_{i} \in \mu} q\left(v_{i}\right) \sum_{v_{k} \in V \backslash M} \phi_{i k}(\mathcal{G}) \cdot \log k_{\text {in }}\left(v_{k}\right) .
$$

Assuming equiprobability, the above expression leads to

$$
H(\mathcal{G} \mid \mu)=\frac{1}{|\mu|} \sum_{v_{i} \in \mu} \sum_{v_{k} \in V \backslash M} \phi_{i k}(\mathcal{G}) \cdot \log k_{\text {in }}\left(v_{k}\right) .
$$

\section{Topological richness: Pyramidal condition}

Let us now estimate the topological richness of a causal graph, i.e., the average amount of information needed to describe a given top-down path within the structure. Let us observe that the kind of question we are trying to answer is the same as the one explored above, but considering the topdown approach. Therefore, the mathematical form of this quantity, to be referred as $H(G \mid M)$, will be formally analogous to the previous one, but considering that we are going onward according to the causal flow. Thus,

$$
H(G \mid M)=\frac{1}{m} \sum_{v_{i} \in M} \sum_{v_{k} \in V \backslash \mu} \psi_{i k}(\mathcal{G}) \cdot \log k_{\text {out }}\left(v_{k}\right),
$$

where $m$ is the cardinality of $M$ - the set of maximal nodes. $\psi_{i k}$ is analogous to $\phi_{i k}$ of Eq. 16. In this case, elements $i k$ of matrix $\Psi$ represent the probability to cross node $v_{k}$ departing from $v_{i} \in M$ according to the causal flow. The explicit expression of $\Psi$ is defined from matrix $\mathbf{B}^{\prime}(\mathcal{G})$,

$$
B^{\prime}(\mathcal{G})_{i j}=\frac{A_{i j}(\mathcal{G})}{k_{\text {out }}\left(v_{i}\right)} .
$$

Then,

$$
\psi_{i j}(\mathcal{G})=\sum_{k \leq L(\mathcal{G})}\left(\left[\mathbf{B}^{\prime}\right]^{k}(\mathcal{G})\right)_{i j},
$$

and as above, we have

$$
\psi_{i i}(\mathcal{G})=\left(\left[\mathbf{B}^{\prime}\right]^{0}(\mathcal{G})\right)_{i i}=1 .
$$

\section{Hierarchy}

As we shall see, the above definition of information will bring us the ingredients to define a hierarchy index according to the list detailed in Sec. III. Roughly speaking, what we propose in the following lines is to evaluate the balance between the pyramidal structure of the graph against the degree of reversibility of the paths it generates, i.e., the balance between $H(\mathcal{G} \mid M)$ and $H(\mathcal{G} \mid \mu)$. However, in order to rigorously characterize hierarchy, we need to properly treat the studied graph attending to the different layers of its feedforward structure. The analysis of the graph structure allows us to identify and quantify deviations from the perfect structure at any level of the graph. The starting point will involve the characterization of a layered structure within the graph defining a partition $W$ of the set of nodes.

\section{Dissecting the layer structure}

Given the DAG $\mathcal{G}(V, E)$, let us define two partitions of $V$, $W=\left\{\omega_{1}, \ldots, \omega_{m}\right\}$ and $\tilde{W}=\left\{\tilde{\omega}_{1}, \ldots, \tilde{\omega}_{m}\right\}$. The members of such partitions are the layers of the DAG by either performing a top-down or bottom-up leaf removal algorithm. ${ }^{5,23}$ Specifically, the first members of such partitions are defined as

$$
\omega_{1}=\left\{v_{i} \in V: k_{\text {out }}\left(v_{i}\right)=0\right\}
$$

and

$$
\tilde{\omega}_{1}=\left\{v_{i} \in V: k_{\text {in }}\left(v_{i}\right)=0\right\} .
$$


We observe that $\omega_{1}=\mu$ and that $\tilde{\omega}_{1}=M$. With the above subsets of $V$ we can define the graphs $\mathcal{G}_{1}\left(V_{1}, E_{1}\right)$ and $\tilde{\mathcal{G}}_{1}\left(\tilde{V}_{1}, \tilde{E}_{1}\right)$ in the following ways:

$$
V_{1}=V \backslash \omega_{1} ; \quad E_{1}=E \backslash\left\{\left\langle v_{i}, v_{k}\right\rangle: v_{k} \in \omega_{1}\right\}
$$

and

$$
\tilde{V}_{1}=V \backslash \tilde{\omega}_{1} ; \quad \tilde{E}_{1}=E \backslash\left\{\left\langle v_{i}, v_{k}\right\rangle: v_{k} \in \tilde{\omega}_{1}\right\} .
$$

respectively. Similarly, we build $\omega_{2}, \ldots, \omega_{|W|}$ as

$$
\begin{aligned}
\omega_{2} & =\left\{v_{i} \in V_{1}: k_{\text {out }}\left(v_{i}\right)=0\right\}, \\
\omega_{|W|-2} & =\left\{v_{i} \in V_{|W|-1}: k_{\text {out }}\left(v_{i}\right)=0\right\},
\end{aligned}
$$

and

$$
\begin{gathered}
\tilde{\omega}_{2}=\left\{v_{i} \in \tilde{V}_{1}: k_{\text {in }}\left(v_{i}\right)=0\right\}, \\
\tilde{\omega}_{|W|}=\left\{v_{i} \in \tilde{V}_{|W|-2}: k_{\text {in }}\left(v_{i}\right)=0\right\},
\end{gathered}
$$

respectively. We therefore defined two sequences of subgraphs of $\mathcal{G}$ ordered by inclusion, namely,

$$
\mathcal{G}_{|W|-1} \subseteq \cdots \subseteq \mathcal{G}_{1} \subseteq \mathcal{G}
$$

and

$$
\tilde{\mathcal{G}}_{|W|-1} \subseteq \cdots \subseteq \tilde{\mathcal{G}_{1}} \subseteq \mathcal{G},
$$

where it is easy to observe that

$$
\mathcal{G}_{|W|-1}=M \quad \text { and } \quad \tilde{\mathcal{G}}_{|W|-1}=\mu .
$$

In Fig. 3 we describe the generation of these subsets of graphs for a given toy model of a DAG. In summary, we constructed two collections of subsets by finding the layered structure using a bottom-up leaf removal algorithm (pruning the elements having $k_{\text {out }}=0$ successively) and a top-down leaf removal algorithm (i.e., pruning the elements having $k_{\text {in }}$ $=0$ ). Note that, even if

$$
|W|=|\tilde{W}|,
$$

one cannot assume

$$
w_{i}=\tilde{w}_{|W|-i},
$$

except in the symmetrical cases.

\section{The hierarchy index}

In order to generate a normalized estimator $f(\mathcal{G})$ (between -1 and 1) accounting for the balance between $H(\mathcal{G} \mid M)$ and $H(\mathcal{G} \mid \mu)$, we define it as

$$
f(\mathcal{G}) \equiv \frac{H(\mathcal{G} \mid M)-H(\mathcal{G} \mid \mu)}{\max \{H(\mathcal{G} \mid M), H(\mathcal{G} \mid \mu)\}} .
$$

Because both the layered structure and its pyramidal composition must be taken into account, the hierarchical index of the graph must be weighted taking into account the successive layers of the system. This avoids to identify as completely hierarchical those structures not perfectly satisfying the pyramidal condition. Therefore, the hierarchical index of a feedforward net, to be indicated as $v(\mathcal{G})$, will be the average among the $|W|-2$ subgraphs $\mathcal{G}_{1}, \ldots, \mathcal{G}_{k}, \ldots$, the $|W|-2$ subgraphs $\tilde{\mathcal{G}_{1}}, \ldots, \tilde{\mathcal{G}_{k}}, \ldots$, and $\mathcal{G}$ itself-note that we average between $2|W|-3$ objects, i.e.,

$$
v(\mathcal{G})=\frac{1}{2|W|-3}\left(f(\mathcal{G})+\sum_{i<|W|-1} f\left(\mathcal{G}_{i}\right)+f\left(\tilde{\mathcal{G}_{i}}\right)\right) .
$$

It is strictly necessary to take into account all these subgraphs in order to identify any violation of the hierarchy conditions at any level of the structure. We can go a step further by imposing symmetry in the pyramidal structure as suggested above, to distinguish among different topologies such as those displayed in Fig. 2(a). Let us indicate by $\Pi_{M \mu}\left(\mathcal{G}_{k}\right)$ the set of paths from $M$ to $\mu$ present in the graph $\mathcal{G}_{k}$. The socalled Jensen's inequality ${ }^{6}$ provides an upper bound of the information content, which, in our case reads

(i) $\quad H\left(\mathcal{G}_{k} \mid M\right) \leq \log \left|\Pi_{M \mu}\left(\mathcal{G}_{k}\right)\right|$,

(ii) $\quad H\left(\mathcal{G}_{k} \mid \mu\right) \leq \log \left|\Pi_{M \mu}\left(\mathcal{G}_{k}\right)\right|$.
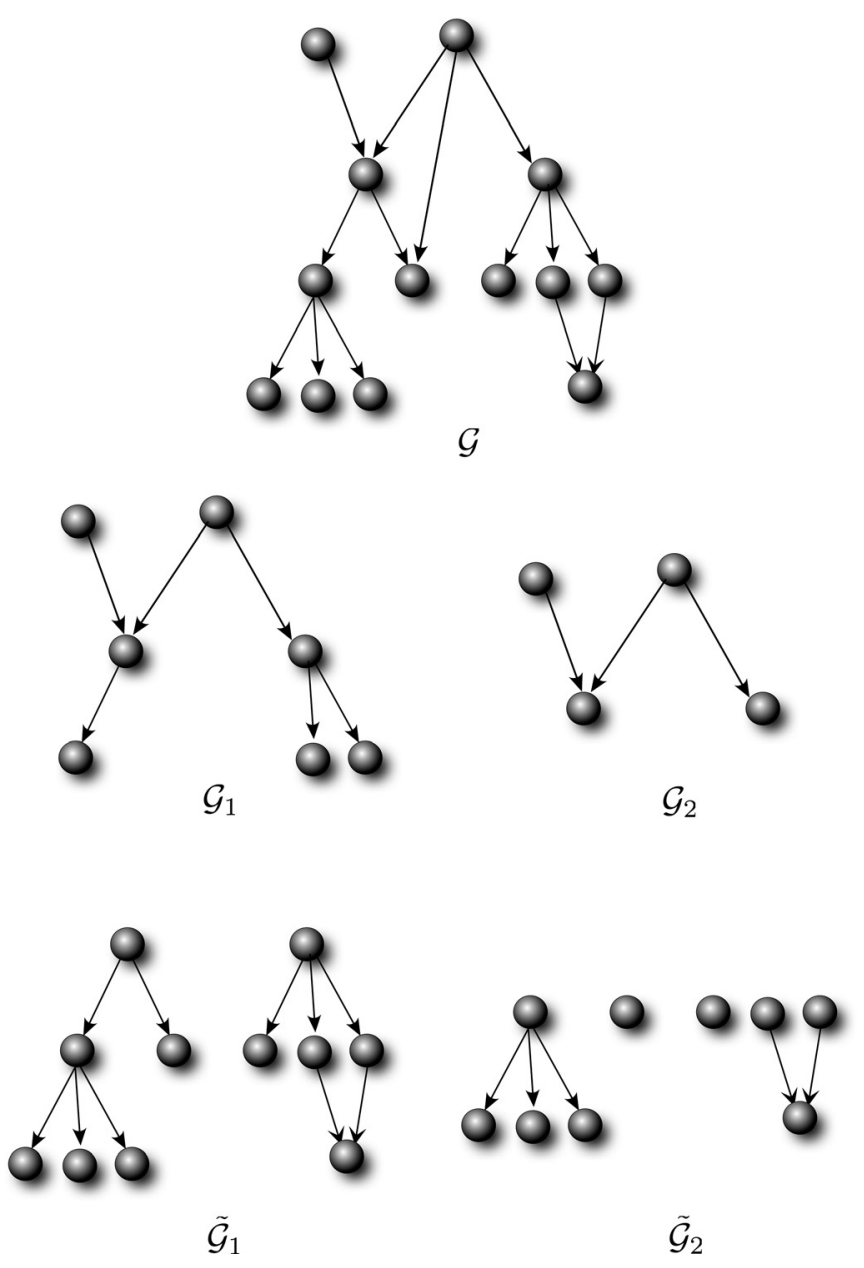

FIG. 3. How to obtain the different subgraphs $\mathcal{G}, \mathcal{G}_{1}, \mathcal{G}_{2}, \widetilde{\mathcal{G}_{1}}, \widetilde{\mathcal{G}_{2}}$ involved in the evaluation of hierarchy. $\mathcal{G}_{1}$ and $\mathcal{G}_{2}$ are obtained through successive application of bottom-up leaf removal algorithm, which implies that we remove all nodes having $k_{\text {out }}=0 . \widetilde{\mathcal{G}_{1}}$ and $\widetilde{\mathcal{G}_{2}}$ are obtained by the successive application of a top-down leaf removal algorithm, thereby removing the nodes having $k_{\text {in }}=0$. We observe that the generation of $\widetilde{\mathcal{G}_{1}}$ and $\widetilde{\mathcal{G}_{2}}$ implies the breaking of the net-see text. 
We observe that (a) is only achieved when all $\left|\Pi_{M \mu}\right|$ from $M$ to $\mu$ are equiprobable, being this equiprobability an indicator of symmetry. The same applies to (b), but now we consider the bottom-up estimator, when paths are considered from $\mu$ to $M$. In this case, attending to the symmetric condition we can define and estimator analogous to $f$, namely,

$$
g(\mathcal{G})=\frac{H\left(\mathcal{G}_{k} \mid M\right)-H\left(\mathcal{G}_{k} \mid \mu\right)}{\log \left|\Pi_{M \mu}(\mathcal{G})\right|} .
$$

Accordingly, the symmetrized version of the hierarchy index, $v_{s}(\mathcal{G})$, would be

$$
v_{s}(\mathcal{G})=\frac{1}{2|W|-3}\left(g(\mathcal{G})+\sum_{i<|W|-1} g\left(\mathcal{G}_{i}\right)+g\left(\mathcal{G}_{i}\right)\right) .
$$

To ensure the consistency of $v$ and $v_{s}$, we must overcome the last conceptual problems. When performing the leaf removal operation one can break the graph in many connected components having no connections among them. Let us indicate as $\mathcal{C}_{1}(i), \ldots, \mathcal{C}_{k}(i)$ the set of components of our graph $\mathcal{G}_{i}$, each of them obtained from the set $V_{k}(i) \subseteq V_{i}$ of nodes and theirs. In this case, the natural way to proceed is to average the individual contributions of the different connected components of $\mathcal{G}_{i}$ or $\tilde{\mathcal{G}}_{i}$ according to the number of nodes they have against $\left|V_{i}\right|$ or $\tilde{V}_{i}$, leading to

$$
f\left(\mathcal{G}_{i}\right) \equiv \frac{1}{\left|V_{i}\right|} \sum_{\mathcal{C}_{k}(i)}\left|V_{k}(i)\right| f\left(\mathcal{C}_{k}(i)\right)
$$

The same applies to $g\left(\mathcal{G}_{i}\right)$ for the computation of $v_{s}$. Finally, we impose, for both mathematical and conceptual consistency that

$$
(\max \{H(\mathcal{G} \mid M), H(\mathcal{G} \mid \mu)\}=0) \Rightarrow(v(\mathcal{G}) \equiv 0) .
$$

Furthermore, if $E=\emptyset$ (i.e., the case where the graph consists of a single node),

$$
v(\mathcal{G}) \equiv 0 .
$$

According to this formulation, some scenarios would lead to $v(\mathcal{G})=0$. The simplest one is the just mentioned by definition, consisting of a single node. Another one is the linear feedforward chain having two or more linked nodes. It is clear that in these cases $H(\mathcal{G} \mid M)=H(\mathcal{G} \mid \mu)=0$. It is worth to stress that this particular situation matches with the causal graph of a total order relation, and therefore, we have the way to differentiate this particular graph from other structures having null hierarchy. Finally, a third class of structures belongs to the family of nonhierarchical graphs. They give $v(\mathcal{G})=0$ because $H(\mathcal{G} \mid M)=H(\mathcal{G} \mid \mu)$. This is the case of Erdös Rényi (ER) DAGs or DAG cliques. In these cases, the causal graph is not hierarchical because all the diversity of paths generated when crossing the causal flow downward is neutralized by the uncertainty in recovering any causal path backward.

\section{Numerical exploration}

In this section, we evaluated the hierarchy of several toy models in order to intuitively grasp the scope of the measure. In Fig. 4, we evaluated the hierarchy index (both the raw one and the symmetrical one) for several structures, leading to hierarchical, antihierarchical, and nonhierarchical structures. The figure illustrates the impact of number of maximals and minimals and the multiplicity of pathways in relation to the existence of a pyramidal and predictable structure. We observe that deviations from tree and inverted tree configurations lead to a nonbinary interpretation of hierarchy.

Furthermore, we measured (Fig. 5) the impact in terms of hierarchy of arc addition preserving the acyclic character. Staring from the two extreme tree graphs (the feedforward and the inverted ones, respectively), we add arcs at random until we reach a fully connected feedforward structure in both situations. We consider the starting point of our numerical experiment a binary tree, $\mathcal{T}(V, E)$ containing $n=15$ nodes. We construct an inverted binary tree $\mathcal{T}^{\prime}(V, E)$ by the transposition of the adjacency matrix of $\mathcal{T}(V, E)$. In both graphs we say that, consistently with the ordering property of DAGs, given an $\operatorname{arc}\left(\left\langle v_{i}, v_{j}\right\rangle \in E\right)$ then $(i<j)$. In an iterative process we construct two new DAGs $\mathcal{G}^{i}\left(V, E^{i}\right)$, where $i$ labels the number of additions of new arcs to the underlying $\mathcal{T}(V, E)$ and $\mathcal{T}^{\prime}(V, E)$. The process ends when graphs achieve the directed acyclic clique condition, i.e., the linearly ordered graph $\mathcal{G}^{*}=\left(V, E^{*}\right)$ containing 15 nodes.

$$
\left(\forall v_{i}, v_{j} \in V\right):(i>j)\left(\left\langle v_{j}, v_{i}\right\rangle \in E^{*}\right) .
$$

\begin{tabular}{|c|c|c|c|c|c|c|c|}
\hline कै & है & की & 14 & $\begin{array}{l}0 \\
1 \\
0 \\
\vdots \\
1 \\
1 \\
0\end{array}$ & 8 & o & $\underset{q}{90} \pm$ \\
\hline $\begin{aligned} \nu(\mathcal{G}) & =1 \\
\nu_{\mathrm{s}}(\mathcal{G}) & =1\end{aligned}$ & $\begin{array}{r}\nu(\mathcal{G})=1 \\
\nu_{\mathrm{s}}(\mathcal{G})=0.989\end{array}$ & $\begin{aligned} \nu(\mathcal{G}) & =0.917 \\
\nu_{\mathrm{s}}(\mathcal{G}) & =0.862\end{aligned}$ & $\begin{aligned} \nu(\mathcal{G}) & =0.430 \\
\nu_{\mathrm{s}}(\mathcal{G}) & =0.305\end{aligned}$ & $\begin{aligned} \nu(\mathcal{G}) & =0 \\
\nu_{\mathrm{s}}(\mathcal{G}) & =0\end{aligned}$ & $\begin{aligned} \nu(\mathcal{G}) & =0 \\
\nu_{\mathrm{s}}(\mathcal{G}) & =0\end{aligned}$ & $\begin{aligned} \nu(\mathcal{G}) & =-1 \\
\nu_{\mathrm{s}}(\mathcal{G}) & =-1\end{aligned}$ & $\begin{aligned} \nu(\mathcal{G}) & =-1 \\
\nu_{\mathrm{s}}(\mathcal{G}) & =-1\end{aligned}$ \\
\hline
\end{tabular}

For statistical significance, we performed 250 replicas of the numerical experiment. Then, $\langle v\rangle$ and $\left\langle v_{\mathrm{s}}\right\rangle$ and their respective standard deviations were calculated for each set of iterations. Figure 5 shows that starting from an initial value

FIG. 4. Different values of the hierarchy index corresponding to some toy DAGs. $v(\mathcal{G})$ refers to the hierarchical index where the symmetry is not considered and $v_{\mathrm{s}}(\mathcal{G})$ refers to the hierarchical index where symmetry is taken into account—see text. 


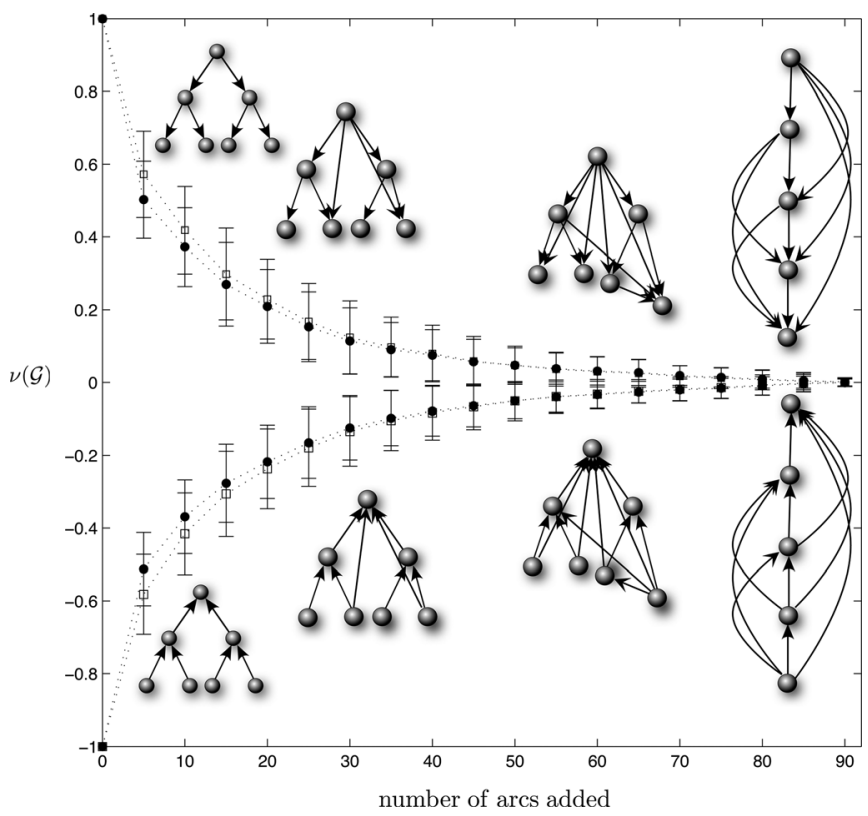

FIG. 5. The evolution of the hierarchy index. We start with a binary tree and a binary inverted tree with $n=15$ and $|E|=14$. For both the graphs we add, in an iterative way, arcs at random until we reach the feed forward clique configuration. Note that the link addition to the two initial treelike structures converges in the same full connected configuration which contains

$\left(\begin{array}{c}15 \\ 2\end{array}\right)$ links. In both the experiments, squares represent $v$ values and triangles $v_{\mathrm{s}}$. As expected, $|v|>\left|v_{\mathrm{s}}\right|$, except in limit cases. The small graphs provide a visual clue for type of structures we are obtaining through the arc enrichment process. For every point in the chart, mean, and standard deviation were calculated from 250 replicas. Entropies were computed considering $\log _{2}$.

of $v=1$ for $\mathcal{T}(V, E)$ and $v=-1$ for $\mathcal{T}^{\prime}(V, E)$, the addition of feedforward arcs causes a decrease in absolute value of the hierarchical indexes until $v=v_{\mathrm{s}}=0$ corresponding to a total linear ordered structure where every possible feedforward path is included in the graph. As expected, $|v|>\left|v_{\mathrm{s}}\right|$ except in the extreme full-connected cases.

We finally test the case of a directed acyclic ER graph $\mathcal{R}(V, E)$. This is an interesting example of a topologically homogeneous DAG with noncorrelation in terms of $k_{\text {in }}$ and $k_{\text {out }}{ }^{9}$ Graphs were obtained by the construction of an undirected ER graph $\mathcal{G}_{\mathrm{ER}}\left(V, E^{u}\right)$, where $E^{u}$ is the set of edges (undirected links). Directed acyclic condition was obtained by a process of random numbering of nodes. ${ }^{9}$ The direction of the arrows was defined attending

$$
\left\langle v_{i}, v_{j}\right\rangle \in E:\left(\left\{v_{i}, v_{j}\right\} \in E^{u} \wedge i<j\right),
$$

[i.e., condition depicted in Eq. 6]. Figure 6 shows a representative behavior of the null hierarchical character of $\mathcal{R}(V, E)$ ensembles. Note that the normal distribution is centered for $v$ and $v_{\mathrm{s}}$ at zero values, indicating that such random structures have not any hierarchical organization.

\section{DISCUSSION}

Hierarchical patterns are known to pervade multiple aspects of complexity. In spite of their relevance, it is not obvious in general how to formalize them in terms of a quanti- tative theory. This paper presents a definition of hierarchy to be applied to the so-called causal graphs, i.e., connected, directed acyclic graphs where arcs depict some direct causal relation between the elements defining the nodes. It is therefore a measure of hierarchy over the structure of the causal flow. The conceptual basis of this measure is rooted in two fundamental features defining hierarchy: the absence of ambiguity in recovering the causal flow and the presence of a pyramidal structure. The hierarchy index presented here weights the deviations from such general properties. The specific expression for this index is derived using techniques and concepts from information theory. It is shown, thus, that the requirements of hierarchy naturally fit the tension between richness in causal paths against the uncertainty in recovering them, depicted by a balance between two conditional entropies.

Under our previous assumptions, we have shown that the feedforward tree is the structure that fully satisfies the conditions for a perfect hierarchical system. Interestingly, the identification of tree structures as perfect representation of hierarchies is a long standing idea. ${ }^{31}$ In this way, our mathematical formalization establishes a bridge between the qualitative idea of hierarchy and its quantification. Our approach allows to measure the hierarchy of any system, provided that it can be represented in a feedforward causal graph.

Throughout the paper, we emphasized that although hierarchy is deeply tied to order, there are strong reasons to go beyond it. The most obvious one is that order is a well-established concept, and therefore, there is no need for the use of a different word, if we identify it with hierarchy. Nevertheless, there is another important issue tied to the intuitive notion of hierarchy that must be taken into account. We propose that hierarchy must feature the pyramidal nature of the connective patterns and that this pyramidal structure must be in agreement with the top-down nature of the feedforward flow of causality.

Information theory reveals to be extremely suitable to define a hallmark to study hierarchy in the terms described in this paper: the richer the structure (but at the same time,
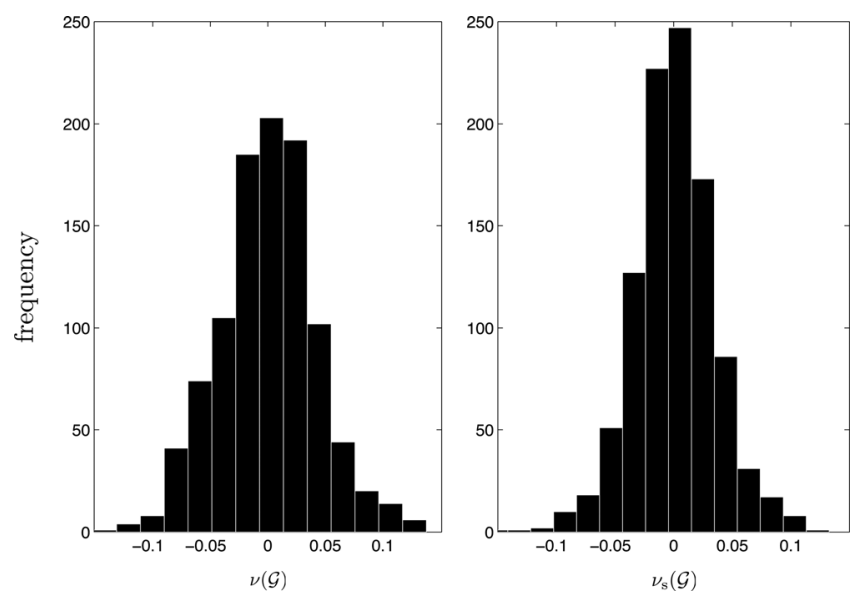

FIG. 6. Distribution of $v$ and $v_{\mathrm{s}}$ for an ensemble of 1,000 replicas of directed acyclic ER graphs of $|V|=500$ and $\langle k\rangle=4$ caption. Numerical results show Gaussian-like distributions centered at zero. Note that $v_{\mathrm{s}}$ distribution displays a narrower variation than the $v$ one in agreement with the $|v| \geq\left|v_{\mathrm{s}}\right|$ inequality. 
reversible, in topological terms), the more hierarchical it is. In this way, because the conditions we defined for a system to be perfectly hierarchical lead us to conclude that a feedforward tree is the perfect hierarchical structure because it maximizes the richness without the loss of predictability. It is worth to note that precisely the pyramidal condition is the key point to guarantee the predictability. The extreme case is the feedforward clique-see Fig. 4. Although richness can be increased through the pathway redundancy, this effect cancels out due to decreased predictability, leading to a nonhierarchical structure. A particular case is the linear chain. This representation of a total order relation has null values for both entropies. In other words, a perfect predictable system but without richness. It is worth to note that both the cases are not pyramidal structures. By contrast, antihierarchical ones exhibit an inverted pyramidal structure, leading to a different effect. From the perspective of our formalism, the antihierarchical organization occurs by minimizing richness and predictability. In consequence, other structure different from an inverted tree will be less antihierarchical. Therefore, it is easy to see that the hierarchical index in absolute value measures the closeness of an (anti)hierarchical tree structure, capturing somehow the path complexity of the structure.

Further work should explore the relation of this theoretical achievement within the framework of a formal measure of complexity. Additionally, this research could be expanded to a more general class of directed graphs containing cycles. This latter point would be achieved by properly defining a measure of how well-ordered is a net, for it is clear that the presence of cycles will generate conceptual problems in the identification of the causal flow.

\section{ACKNOWLEDGMENTS}

This work was supported by the EU 6th framework project ComplexDis (NEST-043241, C.R.-C. and J.G.), the UTE project CIMA (J.G.), Fundación Marcelino Botín (C.R.-C.), the James McDonnell Foundation (B.C.-M. and R.V.S.), and the Santa Fe Institute (R.V.S.). We thank Complex System Lab members for fruitful conversations and an anonymous reviewer for his/her constructive suggestions.

\footnotetext{
${ }^{1}$ Ash, R. B., Information Theory (New York, Dover, 1990).

${ }^{2}$ Bassett, D. S., Bullmore, E., Verchinski, B. A., Mattay, V. S., Weinberger, D. R., and Meyer-Lindenberg, A., "Hierarchical organization of human cortical networks in health and schizophrenia," J. Neurosci. 28, 9239 (2008).

${ }^{3}$ Clauset, A., Moore, C., and Newman, M. E. J., "Hierarchical structure and the prediction of missing links in networks," Nature 453, 98 (2008).

${ }^{4}$ Corominas-Murtra, B., Rodriguez-Caso, C., Goñi, J., and Solé, R. V., "Topological reversibility and causality in feed-forward networks," New J. Phys. 12, 32767, (2010).

${ }^{5}$ Cosentino-Lagomarsino, M., Jona, P., Bassetti, B., and Isambert, H., "Hierarchy and feedback in the evolution of the escherichia coli transcription network," Proc. Natl. Acad. Sci. USA 104, 5516 (2007).

${ }^{6}$ Cover, T. M., and Thomas, J. A., Elements of Information Theory (John Wiley and Sons, New York, 1991).

${ }^{7}$ Eldredge, N., Unfinished synthesis: Biological Hierarchies and Modern Evolutionary Thought (Oxford University Press, New York, 1985).
}

${ }^{8}$ Estrada, E., "Information mobility in complex networks," Phys. Rev. E 80, 026104 (2009).

${ }^{9}$ Goñi, J., Corominas-Murtra, B., Solé, R. V., and Rodríguez-Caso, C., "Exploring the randomness of directed acyclic networks," Phys. Rev. E 82, 066115 (2010).

${ }^{10}$ Gross, J., and Yellen, J., Graph Theory and Its Applications (CRC, Boca Raton, Florida, 1998).

${ }^{11}$ Hirata, H., and Ulanowicz, R., "Information theoretical analysis of the aggregation and hierarchical structure of ecological networks," J. Theor. Biol. 116, 321 (1985).

${ }^{12}$ Huberman, B. A., and Hogg, T., "Complexity and adaptation," Phys. D 22, 376 (1986).

${ }^{13}$ Khinchin, A. I., Mathematical Foundations of Information Theory (Dover Publications, 1957).

${ }^{14}$ Kolars, J. F., and Nystuen, J. D., Human Geography: Spatial Design in World Society (McGraw-Hill, New York, 1974).

${ }^{15}$ Kuratowski, C., "sur la notion de l'ordre dans la théorie des ensembles," Fundam. Math. 2, 161 (1921).

${ }^{16} \mathrm{Ma}$, H.-W., Buer, J., and Zeng, A.-P., "Hierarchical structure and modules in the escherichia coli transcriptional regulatory network revealed by a new top-down approach," BMC Bioinf. 5, 199 (2004).

${ }^{17} \mathrm{McShea}, \mathrm{D}$. W., "The hierarchical structure of organisms," Paleobiology 27, 405 (2001).

${ }^{18}$ Meunier, D., Lambiotte, R., Ersche, A. F. K., and Bullmore, E. T., "Hierarchical modularity in human brain functional networks," Front Neuroinf. 3, 37 (2009).

${ }^{19}$ Miller, W., "The hierarchical structure of ecosystems: Connections to evolution," Evo. Edu. Outreach 1, 16 (2008).

${ }^{20}$ Pattee, H. H., Hierarchy Theory: The Challenge of Complex Systems (G. Braziller, New York, 1973).

${ }^{21}$ Rammal, R., Toulouse, G., and Virasoro, M., "Ultrametricity for physicists," Rev. Mod. Phys. 58, 765 (1986).

${ }^{22}$ Ravasz, E., Somera, A. L., Mongru, D. A., Oltvai, Z. N., and Barabási, A. L., "Hierarchical organization of modularity in metabolic networks," Science 297, 1551 (2002).

${ }^{23}$ Rodríguez-Caso, C., Corominas-Murtra, B., and Solé, R. V., "On the basic computational structure of gene regulatory networks," Mol. Biosyst. 5, 1617 (2009).

${ }^{24}$ Sales-Pardo, M., Guimerà, R., Moreira, A. A., and Amaral, L. A. N., "Extracting the hierarchical organization of complex systems," Proc. Natl. Acad. Sci. USA 104, 15224 (2007).

${ }^{25}$ Shannon, C. E., "A mathematical theory of communication," Bell Syst. Tech. J. 27, 379 (1948).

${ }^{26}$ Simon, H. A., "The architecture of complexity," Proc. Am. Philos. Soc. 106, 467 (1962).

${ }^{27}$ Suppes, P., Axiomatic Set Theory (Dover, New York, 1960).

${ }^{28}$ Trusina, A., Maslov, S., Minnhagen, P., and Sneppen, K., "Hierarchy measures in complex networks," Phys. Rev. Lett. 92, 178702 (2004).

${ }^{29}$ Valverde, S., and Solé, R. V., "Self-organization versus hierarchy in opensource social networks,” Phys. Rev. E: Stat. Nonlin. Soft Matter. Phys. 76, 046118 (2007).

${ }^{30}$ Vázquez, A., Pastor-Satorras, R., and Vespignani, A., "Large-scale topological and dynamical properties of the internet," Phys. Rev. E: Stat. Nonlin. Soft Matter. Phys. 65, 066130 (2002).

${ }^{31}$ Whyte, L. L., Wilson, A. G., and Wilson, D. M., Editors, Hierarchical Structures (Elsevier, New York, 1969).

${ }^{32} \mathrm{Yu}, \mathrm{H}$., and Gerstein, M., "Genomic analysis of the hierarchical structure of regulatory networks,” Proc. Natl. Acad. Sci. USA 103, 14724 (2006).

${ }^{33}$ We observe that this definition, although circular, captures the idea that a hierarchical system displays a kind of regularity that is repeated along the different scales of the system.

${ }^{34}$ Antihierarchy is not a new concept in the field of complex networks (Ref. 28). As we shall see, the hierarchical index is calculated by a difference of two entropy measures, and thus, hierarchical index allows negative and positive values. In this context, terms such as hierarchy, antihierarchy, and nonhierarchy (or alternatively positive, negative, or null hierarchy) could be interpreted with the same meaning as it occurs in the Pearson coefficient where positive correlation and negative correlation (anticorrelation) are opposite tendencies in contraposition with the null tendency of a noncorrelation. 